\title{
Long-Term Complete
} Remission with nab-Paclitaxel, Bevacizumab, and Gemcitabine Combination Therapy in a Patient with Triple-Negative Metastatic Breast Cancer

\author{
Alberto Montero Stefan Glück \\ Division of Hematology/Oncology, Department of Medicine, Sylvester \\ Comprehensive Care Center, University of Miami, Leonard M. Miller School of \\ Medicine, Miami, Fla., USA
}

\section{Key Words}

Triple-negative breast cancer - Metastatic breast cancer - HER2 negative · nab-paclitaxel $\cdot$ Long-term response

\begin{abstract}
This is a case study of a 52-year-old female patient diagnosed in June 2007 with primary metastatic invasive ductal carcinoma of the left breast and synchronous metastases in the bone, lymph nodes, and lung. Biopsy results of the tumor tissue were negative for the estrogen receptor, progesterone receptor, and human epidermal growth factor receptor 2 (HER2). In November 2007, she participated in a phase II study of metastatic HER2-negative breast cancer. Treatment consisted of systemic chemotherapy with gemcitabine 1,500 $\mathrm{mg} / \mathrm{m}^{2}$, nab-paclitaxel $150 \mathrm{mg} / \mathrm{m}^{2}$, and bevacizumab $10 \mathrm{mg} / \mathrm{kg}$ once every other week. The patient experienced pain relief in her sternum after 5 weeks of chemotherapy, and her analgesic therapy was discontinued. After 7 months, the patient achieved a complete radiographic response, which was maintained for nearly 2 additional years. She continued receiving treatment throughout this period, requiring 1 dose reduction due to fatigue. The patient experienced no other adverse events, including neuropathy, and continued working uninterrupted throughout her treatment. The patient was discontinued from the study in May 2010 after disease progression, almost a full 3 years after diagnosis. The patient showed minimal response to subsequent therapies but had disease stabilization and died from her disease in April 2012. Median overall survival for patients with metastatic triple-negative breast cancer is between 12 and 13.3 months. This patient survived nearly 5 years following diagnosis. This case exemplifies how therapy with nab-paclitaxel, bevacizumab, and gemcitabine may prolong survival, with minimal toxicity, in select patients with triplenegative metastatic breast cancer.
\end{abstract}




\section{Introduction}

Triple-negative breast cancer (TNBC) is characterized by absence of expression of the estrogen (ER) and progesterone receptors (PR) and no human epidermal growth factor receptor 2 (HER2)/Neu gene amplification [1, 2]. Unlike hormone receptorpositive and HER2-overexpressing breast cancers, TNBC is unresponsive to endocrine therapy and HER2-targeted agents, respectively, thereby limiting available systemic treatment options to conventional cytotoxic chemotherapy $[1,2]$. Patients with TNBC are more likely to have visceral or brain metastases and short relapse-free survival compared with those with other breast cancer subtypes [3-6]. While chemotherapies have been effective for treating early-stage disease, with pathologic complete response (CR) rates exceeding those of hormone receptor-positive subtypes, patients with metastatic disease have shorter disease-free survival progressing rapidly through several lines of chemotherapy. Thus, response to chemotherapy has not translated to improvements in progression-free (PFS) or overall survival (OS) in the metastatic setting, and the overall prognosis for TNBC remains poor [3, 7]. The median OS of patients with metastatic disease is relatively short, with reports between 9 and 13.3 months [3-6]. An unmet clinical need exists for more effective therapies for TNBC, particularly in the metastatic setting [2].

Taxane-based chemotherapy has demonstrated activity in patients with TNBC $[1,2]$. In the phase III Eastern Cooperative Oncology Group 2100 trial in which $91 \%$ of the patients had HER2-negative disease, $51 \%$ of the patients were PR negative and $37 \%$ were ER negative, the addition of bevacizumab to solvent-based paclitaxel demonstrated significantly improved median PFS compared with solvent-based paclitaxel alone (11.8 vs. 5.9 months, respectively); however, this did not translate into a significant improvement in median OS for the combination arm [8]. nab-Paclitaxel, a novel formulation albumin-bound paclitaxel with a mean particle size of $130 \mathrm{~nm}$, was initially designed with the intent to improve the therapeutic index of solvent-based taxanes $[9,10]$. Currently, nab-paclitaxel is indicated for the treatment of breast cancer after failure of combination chemotherapy for metastatic disease or relapse within 6 months of adjuvant chemotherapy [11]. A phase II study examined the combination of $n a b$-paclitaxel, bevacizumab, and gemcitabine as first-line therapy for patients with HER2-negative metastatic breast cancer [12]. Thirteen of 29 patients in this study were also hormone receptor negative. In this small cohort of patients with stage IV TNBC, the clinical benefit rate as defined by CR, partial response, and stabilization of disease was $85 \%$. Additionally, in this study, there were no significant differences in PFS or OS between triple-negative and hormone receptor-positive patients. This work reports on a patient with metastatic TNBC enrolled in this phase II study of nab-paclitaxel, bevacizumab, and gemcitabine who experienced a long-term complete remission lasting just under 2 years and a PFS from diagnosis of nearly 3 years.

\section{Case Report}

In June 2007, a 52-year-old woman was diagnosed with invasive ductal carcinoma. The patient initially presented with chest pain, and a subsequent breast examination revealed a mass in the left breast and a palpable sternal mass. Mammography performed on June 29, 2007, revealed a spiculated 3-cm mass. A lumpectomy and axillary lymph node dissection were performed at an outside institution and results revealed a moderately differentiated, infiltrating ductal carcinoma, high 
nuclear grade, measuring $4 \mathrm{~cm}$ in maximal diameter, with metastases to 2 of 24 axillary nodes. Further staging also revealed sternal and mediastinal metastases, and a computed tomography (CT) scan revealed bilateral pulmonary nodules $(\underline{\mathrm{fig}} \mathbf{1} \mathrm{a}-\mathrm{c})$. Tumor samples were negative for ER and PR, and positive (++) for HER2 by immunohistochemistry. However, fluorescent in situ hybridization results were negative for HER2 gene amplification, and the patient was diagnosed with triple-negative primary metastatic/stage IV breast cancer.

The patient was initially seen at the University of Miami Sylvester Comprehensive Cancer Center and enrolled in the phase II study of nab-paclitaxel, bevacizumab, and gemcitabine for HER2-negative metastatic breast cancer. The patient began therapy on November 21,2007 . She was treated on study with gemcitabine $1,500 \mathrm{mg} / \mathrm{m}^{2}$ by 30 -min infusion, followed by $n a b$-paclitaxel $150 \mathrm{mg} / \mathrm{m}^{2}$ by 30 -min infusion, then bevacizumab $10 \mathrm{mg} / \mathrm{kg}$ by 30 -min infusion once every 2 weeks in a 4 -week cycle. The patient experienced pain relief after 5 weeks on the study (2 infusions). Serial CT scans, performed every 2 months, demonstrated progressive reduction in the size of all target lesions (fig. 1d, e). Following approximately 7 months of treatment, the patient achieved a complete radiographic response in July 2008. She showed a complete clearance of lesions in all imaging studies, which was confirmed on at least 2 separate occasions approximately 2 months apart according to Response Evaluation Criteria In Solid Tumors [13].

Per protocol, the patient was continued on study treatment following remission. One dose reduction, as required by the study protocol, occurred in November 2008 on cycle 12, due to treatment-related fatigue. The dose of gemcitabine was reduced to $1,250 \mathrm{mg} / \mathrm{m}^{2}, n a b$-paclitaxel to $125 \mathrm{mg} / \mathrm{m}^{2}$, with no modification of the bevacizumab dose, and treatment was continued once every 2 weeks on a 4 week cycle. Throughout her treatment, she experienced no major side effects and continued to work uninterrupted except for visits for chemotherapy and evaluation, and her treatment-related fatigue was successfully managed with the dose reduction. The patient did not exhibit any neuropathy.

The patient maintained her response and continued to receive treatment for just over 2 years. After 12 months on study, follow-up was performed every 3 months. In May 2010, the patient had documented disease progression by CT scan. Metastases were found in the lung, bone, and lymph nodes. As specified in the study protocol, the patient discontinued study treatment due to disease progression. She received her last dose of $n a b$-paclitaxel, bevacizumab, and gemcitabine on May 28, 2010.

Three weeks after progressing, the patient received capecitabine at a dose of $2,000 \mathrm{mg} / \mathrm{m}^{2}$ orally twice a day for 2 weeks in 3 -week cycles and showed minor response. However, therapy was stopped due to unacceptable toxicity, including diarrhea, dehydration, and severe palmar plantar erythrodysesthesia. After 2 months, the patient again had documented disease progression. In August 2010 , she initiated therapy with ixabepilone, with a minor radiographic response. From August 2010 to December 2010, she received 8 cycles of treatment with ixabepilone $\left(30 \mathrm{mg} / \mathrm{m}^{2}\right)$ administered intravenously every 3 weeks. In December 2010, the patient requested a chemotherapy holiday. Beginning in November 2011, the patient was treated with eribulin $1.1 \mathrm{mg} / \mathrm{m}^{2}$ intravenously on days 1 and 8 of a 3-week cycle. Treatment was discontinued in March 2012 due to rapid disease progression with development of central nervous system metastases. She was transferred to hospice care where she died a month later.

Throughout the course of her disease, the patient was also treated with supportive therapy for bony metastases. She received zoledronic acid at $4 \mathrm{mg}$ intravenously every 4 weeks beginning in November 2007 when she began the phase II study. In May 2010, she was switched to denosumab $120 \mathrm{mg}$ by subcutaneous injection every 4 weeks. She continued to receive denosumab throughout her chemotherapy holiday from December 2010 to November 2011 until discontinuing therapy in March 2012 after being transitioned to hospice care.

\section{Discussion}

Patients with metastatic TNBC have a very poor prognosis. In a large analysis of 255 patients diagnosed with TNBC at the MD Anderson Cancer Center between 1985 and 
2004 , the median OS of patients with recurrent disease was only 1.0 year $(95 \%$ CI, $0.8-$ 1.2 years) for TNBC patients, significantly shorter than the median OS of 2.3 years $(95 \%$ CI, 1.9-2.7 years) for those with other breast cancer subtypes (HR, 2.5; 95\% CI, 1.8-3.5; $\mathrm{p}<0.001$ ) [3]. Other studies have reported median OS times for patients with recurrent or metastatic TNBC between 9 and13.3 months [4-6]. While median OS has not been specifically assessed in a large patient population initially presenting with metastatic TNBC, it is likely to be similarly poor. Additionally, patients with metastatic TNBC exhibit progressively shorter response durations to successive lines of therapy. In a retrospective analysis of 111 patients with TNBC (14\% of whom presented with metastatic disease at diagnosis), the median duration of response was only 12 weeks (range, 0-73.1 weeks) to first-line therapy, 9 weeks (range, 0-120.9 weeks) to secondline chemotherapy, and 4 weeks (range, $0-59$ weeks) to third-line chemotherapy [5]. There is an unmet clinical need for effective therapies that prolong survival for patients with metastatic TNBC [2].

In the absence of curative therapies for metastatic disease, the goal of therapy is primarily palliative in nature, leveraging systemic treatment with minimal toxicity to both prolong OS and at the same time enhance quality of life by delaying the onset of cancer-related symptoms [14]. Numerous studies have shown that the combination of gemcitabine and a taxane is an effective regimen that is well tolerated with good response rates [15]. The case described here is of a patient with metastatic TNBC treated with first-line combination chemotherapy consisting of $n a b$-paclitaxel, bevacizumab, and gemcitabine every other week. After only 5 weeks and 2 infusions, the patient experienced significant symptom relief from her pain from bone metastases, including the sternum. Following 7 months of treatment, she achieved a complete remission that, with continuous therapy, persisted for nearly 2 years for an overall progression-free period from initial diagnosis of 2 years and 7 months. Following progression, subsequent lines of therapy were able to produce only limited efficacy in disease control. Following her initial progression-free interval of almost 2 years in CR with $n a b$-paclitaxel, bevacizumab, and gemcitabine therapy, subsequent treatment with capecitabine then ixabepilone resulted in short progression-free intervals of only 2 months and 4 months, respectively. While ixabepilone did provide a PFS benefit, it was very modest. From her initial diagnosis in June 2007, the patient survived nearly 5 years, which is greatly improved compared to reported median survival of 9-13.3 months for stage 4 TNBC [3-6].

With the aim to enhance quality of life, systemic treatments with minimal toxicity are preferred for patients with metastatic breast cancer [14]. While the patient did experience treatment-related fatigue while on nab-paclitaxel, bevacizumab, and gemcitabine combination therapy, this was easily managed with a dose reduction. She experienced no other adverse events with this treatment regimen. In addition to a lengthy complete remission, the patient was able to work uninterrupted throughout her treatment period, a factor that may significantly elevate quality of life for many patients.

This patient initially received treatment as a participant in a phase II study of $n a b$ paclitaxel, bevacizumab, and gemcitabine as first-line therapy for HER2-negative metastatic breast cancer [12]. A large proportion (13 of 29) of the patients enrolled in this study had triple-negative disease. Overall, this subset of patients demonstrated promising outcomes, with 38\% achieving a complete radiographic response and $85 \%$ 
experiencing clinical benefit. The overall population showed 28 and 93\% CR and clinical benefit, respectively. Most notably, survival outcomes were almost identical between TNBC patients and those with hormone receptor-positive disease.

Additionally, severe adverse events appeared to be less frequent in the overall study population relative to results in other published chemotherapy studies in patients with metastatic breast cancer.

This case study demonstrates the potential for triplet chemotherapy with nabpaclitaxel, bevacizumab, and gemcitabine to elicit prolonged responses, potentially prolong OS, and improve quality of life for patients with metastatic TNBC. This combination shows promise for the treatment of TNBC and should be the subject of further investigation in larger randomized trials.

\section{Acknowledgements}

Medical writing assistance was provided by Jennifer Leslie, PhD, MediTech Media, funded by Celgene Corporation. The authors are fully responsible for content and editorial decisions for this work.
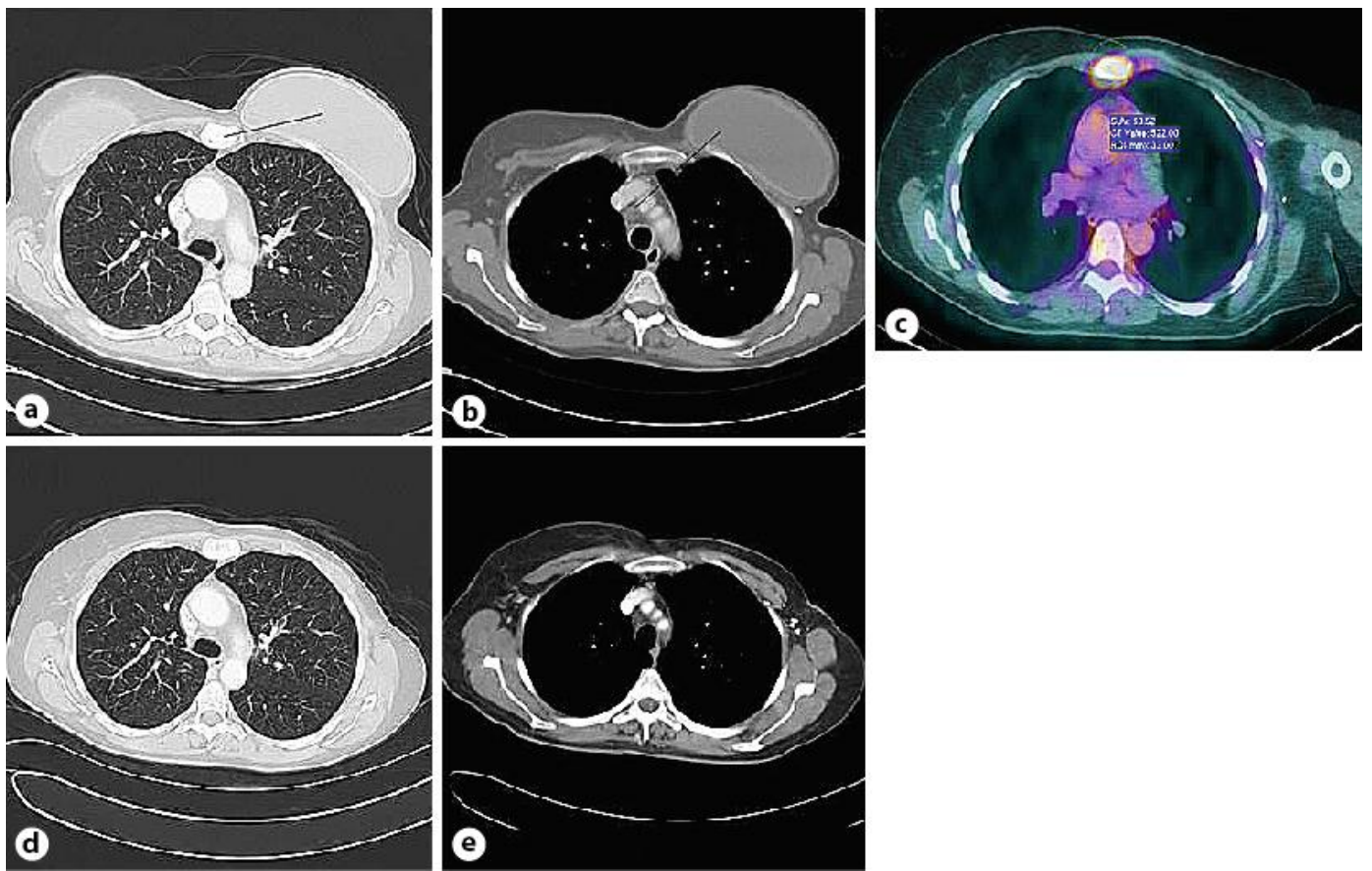

Fig. 1. CT (a, b) and PET (c) scans from November 2007 prior to treatment show sternal and mediastinal metastases. Follow-up imaging on May 2009 (d, e) after approximately 6 months of systemic chemotherapy with $n a b$-paclitaxel, bevacizumab, and gemcitabine combination therapy demonstrating near complete regression of metastatic lesions. 


\section{References}

1 Isakoff SJ: Triple-negative breast cancer: role of specific chemotherapy. Cancer J 2010;16:53-61.

$\checkmark 2$ Hudis CA, Gianni L: Triple-negative breast cancer: an unmet medical need. Oncologist 2011;16(suppl 1):1-11.

-3 Liedtke C, Mazouni C, Hess KR, Andre F, Tordai A, Mejia JA, Symmans WF, Gonzalez-Angulo AM, Hennessy B, Green M, Cristofanilli M, Hortobagyi GN, Pusztai L: Response to neoadjuvant therapy and long-term survival in patients with triple-negative breast cancer. J Clin Oncol 2008;26:1275-1281.

-4 Lin NU, Claus E, Sohl J, Razzak AR, Arnaout A, Winer EP: Sites of distant recurrence and clinical outcomes in patients with metastatic triple-negative breast cancer. Cancer 2008;113:2638-2645.

-5 Kassam F, Enright K, Dent R, Dranitsaris G, Myers J, Flynn C, Fralick M, Kumar R, Clemons M: Survival outcomes for patients with metastatic triple-negative breast cancer: implications for clinical practice and trial design. Clin Breast Cancer 2009;9:29-33

6 Dent R, Trudeau M, Pritchard KI, Hanna WM, Kahn HK, Sawka CA, Lickley LA, Rawlinson E, Sun S, Narod SA: Triple-negative breast cancer: clinical features and patterns of recurrence. Clin Cancer Res 2007;13:4429-4434.

7 Carey LA, Dees EC, Sawyer L, Gatti L, Moore DT, Collichio F, Ollila DW, Sartor CI, Graham ML, Perou CM: The triple negative paradox: primary tumor chemosensitivity of breast cancer subtypes. Clin Cancer Res 2007;13:2329-2334.

-8 Miller K, Wang M, Gralow J, Dickler M, Cobleigh M, Perez EA, Shenkier T, Cella D, Davidson NE: Paclitaxel plus bevacizumab versus paclitaxel alone for metastatic breast cancer. New Engl J Med 2007;357:26662676.

9 Desai NP, Trieu V, Hwang LY, Wu R, Soon-Shiong P, Gradishar WJ: Improved effectiveness of nanoparticle albumin-bound (nab) paclitaxel versus polysorbate-based docetaxel in multiple xenografts as a function of HER2 and SPARC status. Anticancer Drugs 2008;19:899-909.

10 Gradishar WJ, Tjulandin S, Davidson N, Shaw H, Desai N, Bhar P, Hawkins M, O'Shaughnessy J: Phase III trial of nanoparticle albumin-bound paclitaxel compared with polyethylated castor oil-based paclitaxel in women with breast cancer. J Clin Oncol 2005;23:7794-7803.

11 Abraxane $^{\circledast}$ for injectable suspension, (paclitaxel protein-bound particles for injectable suspension) (albumin-bound) [prescribing information]. Summit, NJ: Celegene Corporation, 2012. http://www.abraxane.com/docs/Abraxane_PrescribingInformation.pdf. Accessed June 6, 2012

12 Lobo C, Lopes G, Baez O, Castrellon A, Ferrell A, Higgins C, Hurley E, Hurley J, Reis I, Ri chman S, Seo P, Silva O, Slingerland J, Tukia K, Welsh C, Gluck S: Final results of a phase II study of nab -paclitaxel, bevacizumab, and gemcitabine as first-line therapy for patients with HER2-negative metastatic breast cancer. Breast Cancer Res Treat 2010;123:427-435.

13 Therasse P, Arbuck SG, Eisenhauer EA, Wanders J, Kaplan RS, Rubinstein L, Verweij J, Van Glabbeke M, van Oosterom AT, Christian MC, Gwyther SG: New guidelines to evaluate the response to treatment in solid tumors. J Natl Cancer Inst 2000;92:205-216.

14 National Comprehensive Cancer Network clinical practice guidelines in oncology: breast cancer. Version 2.2012 .

15 Gudena V, Montero AJ, Gluck S: Gemcitabine and taxanes in metastatic breast cancer: a systematic review. Ther Clin Risk Manag 2008;4:1157-1164. 